\title{
Ectodomain Shedding of the Amyloid Precursor Protein: Cellular Control Mechanisms and Novel Modifiers
}

\author{
Stefan F. Lichtenthaler \\ Adolf Butenandt Institute, Ludwig Maximilians University, Munich, Germany
}

\section{Key Words}

Amyloid precursor protein - Alzheimer's disease •

Ectodomain shedding $\cdot$ Secretases $\cdot$ Endocytosis ·

Endophilin

\begin{abstract}
Proteolytic cleavage in the ectodomain of the amyloid precursor protein (APP) is a key regulatory step in the generation of the Alzheimer's disease amyloid- $\beta$ (A $\beta$ ) peptide and occurs through two different protease activities termed $\alpha$ and $\beta$-secretase. Both proteases compete for APP cleavage, but have opposite effects on $A \beta$ generation. At present, little is known about the cellular pathways that control APP $\alpha$-or $\beta$-secretase cleavage and thus $A \beta$ generation. To explore the contributory pathways in more detail we have recently employed an expression cloning screen and identified several activators of APP cleavage by $\alpha$ - or $\beta$-secretase. Among them were known activators of APP cleavage, for example protein kinase $A$, and novel activators, such as endophilin and the APP homolog amyloid precursor-like protein 1 (APLP1). Mechanistic analysis revealed that both endophilin and APLP1 reduce the rate of APP endocytosis and strongly increase APP cleavage by $\alpha$-secretase. This review summarizes the results of the expression cloning screen in the context of
\end{abstract}

\section{KARGER}

Fax +4161306 1234

E-Mail karger@karger.ch

www.karger.com
(C) 2006 S. Karger AG, Basel

$1660-2854 / 06 / 0035-0262 \$ 23.50 / 0$

Accessible online at:

www.karger.com/ndd recent developments in our understanding of the cellular regulation of APP $\alpha$-secretase cleavage. Moreover, it highlights the particular importance of endocytic APP trafficking as a prime modulator of APP shedding.

Copyright $\odot 2006$ S. Karger AG, Basel

\section{Proteolytic Processing of APP}

The amyloid precursor protein (APP) is one of a large number of membrane proteins that are proteolytically converted to their soluble counterparts. This process is referred to as ectodomain shedding and is an important way of regulating the biological activity of membrane proteins [reviewed in 1,2]. The shedding of APP may occur through two different protease activities termed $\alpha$ and $\beta$-secretase, which cleave APP within its ectodomain close to its transmembrane domain [for a review, see 3]. APP cleavage by $\alpha$ - or $\beta$-secretase is a key regulatory process in the generation of the amyloid- $\beta$ (A $\beta$ ) peptide, which is assumed to play an essential role in the pathogenesis of Alzheimer's disease (AD). $\beta$-Secretase, which is the aspartyl protease BACE1, cleaves APP at the N-terminus of the $A \beta$ peptide domain and thus catalyzes the first step in $A \beta$ peptide generation [4]. Subsequently, the 


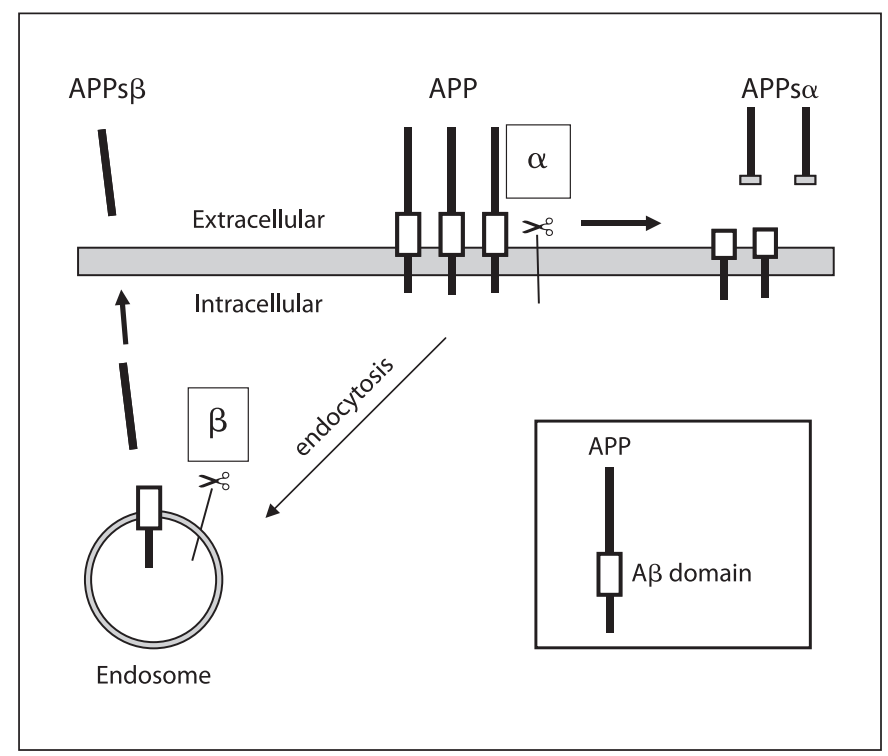

Fig. 1. Ectodomain shedding of APP shedding by $\alpha$ - and $\beta$-secretase. $\alpha$-Secretase cleavage of wild-type APP occurs at or very close to the cell surface, whereas $\beta$-secretase cleavage mainly takes place after endocytosis in the endosomes.

remaining C-terminal APP fragment is cleaved by $\gamma$ secretase within its transmembrane domain at the $\mathrm{C}$-terminus of the $A \beta$ domain, leading to the secretion of the $\mathrm{A} \beta$ peptide [5]. In contrast to $\beta$-secretase, $\alpha$-secretase cleaves within the $A \beta$ sequence, and thereby precludes the generation of the $A \beta$ peptide. $\alpha$-Secretase is a member of the ADAM (a disintegrin and metalloprotease) family of proteases [for a review, see 6]. $\alpha$ - and $\beta$-secretase compete for the ectodomain cleavage of APP [7] (fig. 1), but have opposite effects on $A \beta$ generation. Additionally, $\alpha$-but not $\beta$-secretase generates a secreted form of APP (APPs $\alpha$ ), which has neurotrophic and neuroprotective properties [reviewed in 8]. Thus, shifting APP shedding away from $\beta$ - towards $\alpha$-secretase cleavage may be therapeutically beneficial for $\mathrm{AD}$. In order to do so, it is essential to understand the cellular pathways that regulate the activity of both proteases. At present, little is known about the cellular regulation of BACE1. In contrast, APP $\alpha$-secretase cleavage can be regulated in the cell through different mechanisms, which can be broadly grouped into three categories. First, an activation of distinct intracellular signaling mechanisms or a change in the membrane composition increases APP $\alpha$-shedding [for reviews, see 6,8$]$. Second, changes in APP endocytosis alter $\alpha$-and $\beta$-secretase cleavage, because $\alpha$-secretase cleavage occurs at or very close to the plasma membrane [9], whereas $\beta$-secretase cleavage of APP mainly occurs after endocytosis in the endosomes. Third, the interaction of APP with cytoplasmic adaptor proteins alters APP shedding [for a review, see 10], presumably by affecting APP trafficking and access of APP to the secretases. For all three categories the molecular mechanisms underlying the increase in APP shedding are only partly understood. Thus, we have recently employed expression cloning to explore the contributory cellular pathways systematically and obtained several proteins activating APP shedding $[11,12]$. The identified proteins fall into the three general categories of APP shedding activators described above. This review summarizes their mechanistic analysis in the context of recent developments in our understanding of the cellular regulation of APP $\alpha$-cleavage. This highlights the particular importance of the endocytic trafficking of APP as a prime modulator of APP shedding.

\section{Expression Cloning Screen for Modifiers of APP Shedding}

For the expression cloning screen, a reporter cell line was used that allows measurement of APP shedding in a high-throughput format. For this aim, human embryonic kidney 293 cells were used that stably express a fusion protein consisting of alkaline phosphatase fused to the N-terminus of full-length APP [12]. The 293 cells are a well-established cellular model for the analysis of APP shedding and have the additional advantage that they can be transfected with very high efficiency, which was an essential requirement for the screening approach used. In a 'sib-selection' or 'pool-subdivision' approach, we first used pools of $96 \mathrm{cDNAs}$ from a human brain cDNA library and screened them for activators of APP shedding (fig. 2). Next, to identify the individual cDNA within the pool, which was responsible for activation of APP shedding, the pooled cDNAs were further subdivided and individual cDNAs from that pool were tested for their APP shedding-enhancing activity. With this approach, eight cDNAs were obtained that stimulate the shedding of APP (table 1). They encode protein kinase A (PKA), an N-terminally truncated form of the kinase MEKK2, metabotropic glutamate receptor 3 (mGluR3), endophilins A1 and A3, numblike, an N-terminally truncated form of the palmitoyl-protein thioesterase 1 and the APP homolog amyloid precursor-like protein 1 (APLP1) [11, 12]. cDNAs inhibiting APP secretion were not obtained. Altogether, around 100,000 cDNAs were screened. Considering that cDNA libraries contain many partial cDNAs 
Fig. 2. Expression cloning screen for modulators of APP shedding. The reporter cell line consists of human embryonic kidney 293 cells, which stably express a fusion protein of alkaline phosphatase (AP) and full-length APP. Cleavage of APP by $\alpha$ - or $\beta$-secretase leads to the secretion of the fusion protein into the conditioned medium, where it can be detected and quantified by measuring the alkaline phosphatase reporter enzyme activity. Cells were plated into 96-well plates and transfected with pools of cDNAs. APP fusion protein secretion was measured in all wells. In wells with altered APP secretion, the corresponding cDNA was identified and mechanistically characterized.

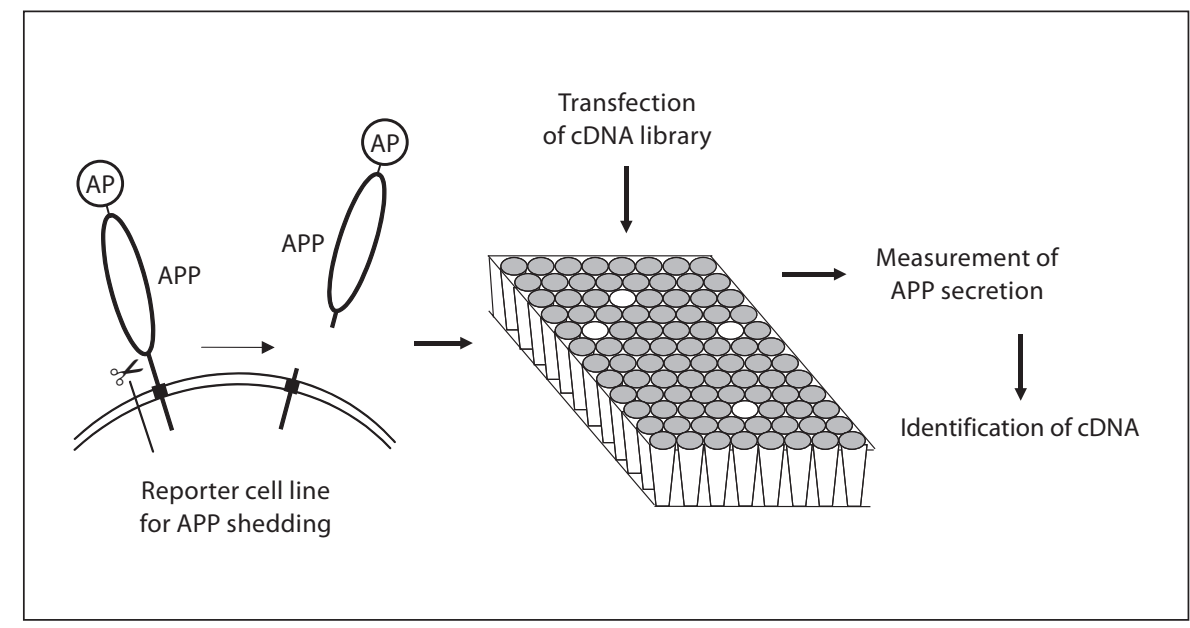

Table 1. Activators of APP shedding obtained by expression cloning

\begin{tabular}{ll}
\hline Protein encoded by cDNA & Full-length or partial cDNA \\
\hline Protein kinase A, catalytic $\alpha$-subunit & full-length \\
MEKK2 (member of the MAPKKK family) & lacking the $5^{\prime}$ end of the coding sequence; encoding an \\
& N-terminally truncated protein \\
mGluR3 & full-length \\
Endophilin A1 & full-length \\
Endophilin A3 & full-length \\
Numblike & full-length \\
Palmitoyl-protein thioesterase 1 & lacking the 5 ' end of the coding sequence; encoding an \\
& N-terminally truncated protein \\
APLP1 & full-length \\
\hline
\end{tabular}

and that cDNAs expressed at high levels are overrepresented in the library, we assume that many more cDNA clones would need to be screened to cover all distinct cDNAs found in the library. The identification of PKA is in agreement with previous publications showing that an activation of PKA by forskolin in rat pheochromocytoma PC12 cells [13] and in human embryonic kidney 293 cells [14] increased APP shedding. This validates the screening approach as it shows that physiologically relevant cDNAs can be obtained.

\section{Specificity of Identified cDNAs for APP Shedding}

Some of the identified cDNAs, such as the endophilins and mGluR3, activated APP shedding in a relatively specific manner, as they had essentially no effect on the shed- ding of unrelated membrane proteins, such as TNF receptor 2 (TNFR2), P-selectin glycoprotein ligand-1 (PSGL-1) or L-selectin [12]. Like APP, all three proteins are subject to ectodomain shedding by ADAM proteases. APLP1 strongly activated shedding of APP but not of L-selectin (fig. 3), demonstrating that APLP1 does not stimulate the shedding of all ADAM protease substrates. Other proteins, such as PKA and the kinase MEKK2, activated the shedding of L-selectin (fig. 3, shown for MEKK2) or of TNFR2 [12] much more strongly than the shedding of APP, showing that they are not specific activators of APP shedding, but instead may contribute to a general cellular program controlling ectodomain shedding. 


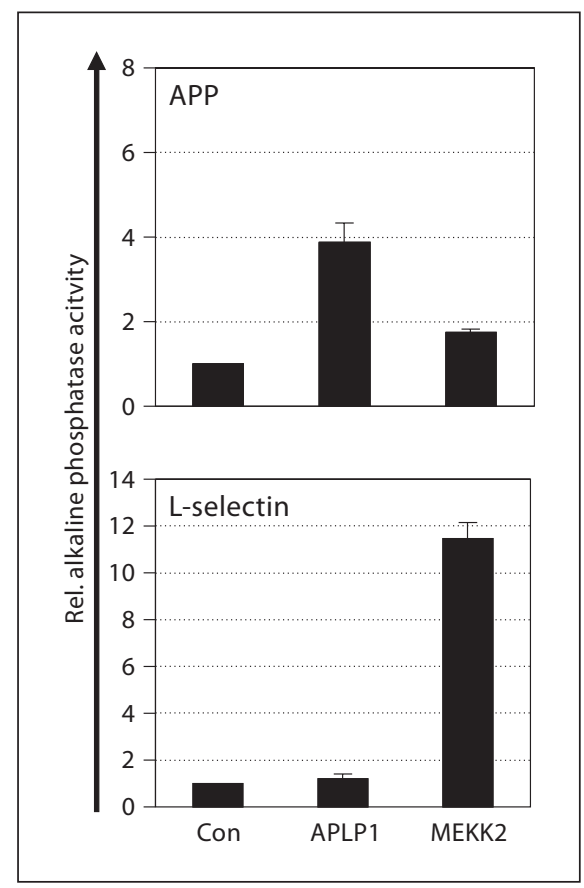

Fig. 3. Specificity of the stimulatory effect of APLP1 on the shedding of APP. Kidney 293 cells stably expressing alkaline phosphatase fusion proteins of APP or L-selectin were transiently transfected with control vector (Con), APLP1 or MEKK2. AP activity was measured in the conditioned medium and represents the mean and standard deviation of two to three independent experiments, each one carried out in duplicate. Alkaline phosphatase activity was normalized to the protein concentration in the cell lysate. The data for APP were part of the set of experiments shown in Neumann et al. [11].

\section{Cellular Control of APP Ectodomain Shedding by Signal Transduction Cascades}

The APP shedding activators PKA and MEKK2 are part of signal transduction pathways, which have previously been shown to control the amount of APP shedding. The $\alpha$-secretase cleavage of APP can be stimulated by MAP kinase signaling, insulin signaling and signaling through PKA or specific $G$ protein-coupled receptors [for reviews see 6,8 ] as well as by calcium [15]. For example, growth factors, such as EGF, or the phorbol ester PMA can activate the MAP kinase cascade and stimulate APP shedding. The molecular and cellular processes activated by these cascades and the mechanisms by which they finally mediate the increase in APP shedding remain largely unknown. Additionally, a diverse group of compounds, such as cholesterol, steroid hormones, nonsteroidal antiinflammatory drugs and cholinesterase inhibitors can modulate APP $\alpha$-secretase cleavage. The underlying molecular mechanisms are partly understood and have been described in more detail elsewhere [for a review, see 6].

One of the proteins identified in the screen, mGluR3, is a novel activator of APP shedding and belongs to the eight-member family of mGluRs. mGluR3 may be particularly interesting for studying APP shedding, as it was one of the cDNAs showing a specific effect on the shedding of APP. Two members of the mGluR family, mGluR1 and mGluR5, have previously been shown to stimulate the secretion of APP [16]. Both mGluRs activate phospholipase $\mathrm{D}$. In contrast, the identified mGluR3 negatively regulates adenylate cyclase and thus, points to a possible role of this separate pathway in the control of APP shedding.

\section{Control of APP Shedding by Modulators of General Endocytosis}

A strong activator of APP shedding identified in the screen was endophilin A3, which belongs to the endophilin family of endocytic and signal transducing proteins [17]. Endophilin consists of a Bin/amphiphysin/Rvs domain, which may be involved in protein dimerization and sensing of membrane curvature, and of a $\mathrm{SH} 3$ domain. Endophilin A3 increased APP shedding even stronger than the metalloprotease ADAM10 [11, 12], which is one of the candidate $\alpha$-secretases for APP. Importantly, we found that endophilin A3 specifically increased APP $\alpha$-secretase cleavage and had no significant effect on $\beta$-secretase cleavage. Mechanistically, endophilin A3 inhibits the rate of APP endocytosis, as measured in a validated anti-APP antibody uptake assay using COS cells cotransfected with APP and either endophilin A3 or GFP as a control $[11,12]$. As a result, more APP becomes available at the cell surface for an increased $\alpha$-secretase cleavage. This fits with previous studies showing that a mutant form of APP, which lacks its cytoplasmic domain including its internalization motif, shows a strong reduction in endocytosis resulting in more APP at the cell surface and increased APP shedding [18]. A strong increase in APP shedding, mainly mediated by $\alpha$-secretase, was also observed for a dominant-negative mutant of the endocytic GTPase dynamin, which inhibits endocytosis of many membrane proteins, including APP $[19,20]$. Conversely, expression of the small $G$ protein Rab5 in murine L1 cells enhances APP endocytosis, resulting in an increased APP cleavage by $\beta$-secretase, in increased $A \beta$ generation and in abnormally enlarged endosomal structures [21]. 
Expression of endophilin A3 not only inhibited the endocytosis of APP but also the endocytosis of fluorescently labeled transferrin [12], revealing that endophilin A 3 is a general, negative regulator of endocytosis, potentially similar to mutants of the endocytic GTPase dynamin. Despite its more general role in endocytosis, the endophilin A3 had a strong effect only on APP shedding but no or only a minor effect on the shedding of other membrane proteins, such as TNFR2 or PSGL-1 [12]. This suggests that APP stands out among other shedding substrates in that its shedding is particularly sensitive to changes in the rate of endocytosis. At present, it is unclear why this is so. Potentially, the endocytosis of TNFR2 and PSGL-1 is regulated differently than the endocytosis of APP or may have a different time course.

\section{APP Interactors Influence APP Shedding}

APP is at the center of a complex protein-protein interaction network involving cytoplasmic adaptor and transmembrane proteins, but the functional role of these interactions is only partly understood. Most of the cytoplasmic interactors seem to compete for the same binding site at or around the conserved GYENPTY motif in the cytoplasmic tail of APP [for recent reviews on APP interactors, see 10, 22]. For example, FE65, X11, and JIP have been shown to bind to this motif and to alter APP shedding, revealing that a change in the interaction of APP with its binding partners is a way to modulate APP shedding. FE65 and X11 have been best studied among the interactors and have opposite effects on APP cleavage. X11 decreases APP shedding, presumably by retaining APP in early compartments of the secretory pathway. In contrast, FE65 stimulates APP shedding. A specific mechanism of how FE65 controls the shedding of APP, but not of unrelated membrane proteins, has been put forward by us and several other groups using different experimental approaches [11, 23-27]. According to this model, FE65 links APP to the LDL receptorrelated protein (LRP; fig. 4A), which is a multifunctional cell surface receptor for proteins involved in lipoprotein metabolism [28]. Formation of the APP-FE65-LRP complex allows efficient endocytosis of APP (fig. 4A). In contrast, disruption of the complex leads to a reduction in APP endocytosis, resulting in an accumulation of APP at the cell surface, where it undergoes increased $\alpha$-secretase cleavage and reduced $\beta$-secretase cleavage (fig. 4B). This is the case in LRP-deficient cells or upon RNAi-mediated knockdown of FE65 [23, 24, 29]. Like-

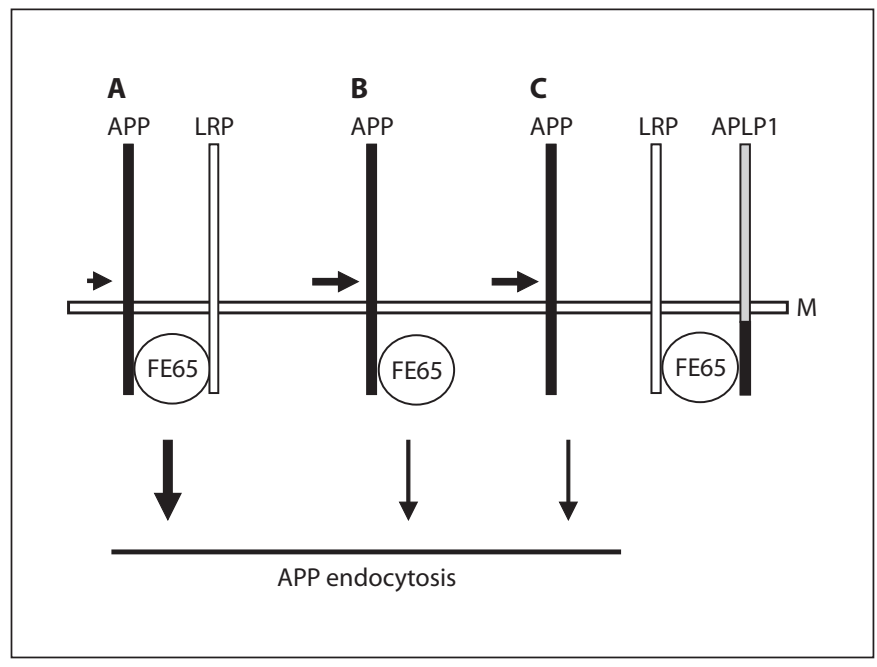

Fig. 4. Model for the APP-FE65-LRP complex. A In LRP expressing wild-type cells APP, FE65 and LRP form a complex, allowing efficient APP endocytosis (bold vertical arrow) and resulting in low levels of APP shedding (thin horizontal arrow). Size of proteins is not drawn to scale. B In LRP-deficient cells (LRP-/-), endocytosis of APP is reduced and APP shedding is increased (bold horizontal arrow). C In cells transfected with APLP1, LRP preferentially forms a complex with APLP1, resulting in APP not being complexed to LRP. This results in a state resembling LRP deficiency (B) and an increase in APP shedding (bold horizontal arrow). $\mathrm{M}=$ Membrane. Figure adapted from Neumann et al. [11].

wise, overexpression of FE65 results in the disruption of the complex, presumably by leading to APP-FE65 complexes and to separate FE65-LRP complexes. Both kinds of complexes lack the third binding partner and therefore are not functional with regard to APP endocytosis.

\section{APLP1 Modulates APP Shedding}

An essential validation of the model described above came from work that we carried out in collaboration with Claus Pietrzik and Christian Haass [11]. We found that the APP-FE65-LRP complex can also be disrupted when proteins are expressed that can functionally replace APP in terms of complex formation with FE65 and LRP, leaving APP without its binding partners (fig. 4C). This happens when the two homologs of APP, APLP1 and APLP2, are expressed. A detailed mechanistic analysis revealed that APLP1 expression reduces APP endocytosis, strongly increases APP $\alpha$-secretase cleavage and reduces APP $\beta$-secretase cleavage [11]. Moreover, the APLP1 effect on 
APP shedding is only observed in LRP-expressing cells, but not in LRP knock-out cells, showing that this effect is LRP dependent. Additionally, mutational analysis revealed that the FE65-binding motif in APLP1 needs to be present in order to increase APP shedding. Importantly, proteins that do not bind FE65 did not affect APP shedding, consistent with the APP-FE65-LRP complex being required for APP endocytosis and shedding. Together, these experiments raise the possibility that changes in the expression levels of the APP homologs APLP1 and APLP2 may influence the shedding of APP. In fact, expression levels of APLP1, APLP2 and even of APP are differentially regulated upon physiological and pathophysiological stimuli, such as during embryonic development, neuronal migration and wound repair [30, 31]. These stimuli may in turn alter the amount of APP shedding. Future studies need to show, whether the complex only consists of the three proteins APP-FE65-LRP, or whether it is part of a multi-protein complex. Given that FE65 consists of several protein-protein interaction domains, it is likely that FE65 may link the complex to other proteins. In fact, FE65 colocalizes with APP in actin-rich lamellipodia in neuronal growth cones [32] and may link APP to cellular motility [33].

\section{Modulation of APP Shedding by the Transmembrane Proteins LRP1B and BRI2}

Interestingly, a homolog of LRP, LRP1B, may form a similar complex with APP as LRP itself. A recent study showed that LRP1B can also be coimmunoprecipitated with APP [34]. It remains to be established whether this interaction is also mediated by FE65. In contrast to LRP, which is rapidly endocytosed and mediates efficient APP endocytosis, LRP1B is very slowly endocytosed. LRP1B reduced APP endocytosis and again increased APP shedding by $\alpha$-secretase [34]. Presumably, LRP1B forms a complex with APP at the expense of LRP, similar to APLP1, which forms the complex with LRP at the expense of APP. These experiments reinforce the notion that changes in the rate of APP endocy tosis determine the amount of APP $\alpha$-secretase cleavage.

Besides LRP and LRP1B, two novel transmembrane interactors of APP have recently been described. Sorting protein-related receptor (SorLa) is a type I transmembrane protein of unknown function, which is expressed in neurons and was shown to coimmunoprecipitate with APP [35]. The other protein is BRI2, which is the first type II membrane protein shown to coimmunoprecipi-

Cellular Control of APP Shedding tate with APP $[36,37]$. Again, the function of BRI2 is unknown, but mutant forms of BRI2 have been linked to dementia and cerebellar ataxia in Danish and British kindreds. The name BRI seems to be derived from the British origin [38]. Currently, it is unclear, whether the interaction between APP and both novel proteins, SorLA and BRI2, occurs directly or is mediated by adapter proteins, as it is the case for LRP. Interestingly, expression of both SorLa and BRI2 strongly inhibited APP $\alpha$-secretase cleavage and also $A \beta$ generation. Although the underlying mechanisms remain to be established in detail, both proteins seem to retain APP in early cellular compartments of the secretory pathway, where APP cannot reach the secretases.

A surprising additional membrane protein interactor has recently been suggested: APP itself. Soba et al. [39] proposed that APP can dimerize in cis and in trans at the cell surface. It will be interesting to see in future studies, whether the dimerization status of APP can influence APP shedding, potentially by modulating the formation or the endocytosis of the APP-FE65-LRP complex.

\section{Conclusion}

Recently, we and others have described several novel modulators of APP trafficking and processing. Although we are only beginning to understand the underlying mechanisms, it becomes more and more clear that not only signaling cascades, changes in membrane composition and interaction of APP with cytoplasmic adaptors (discussed above in the first paragraph) but also changes in APP trafficking, and specifically in the rate of APP endocytosis, can have a major effect on APP processing by $\alpha$-and $\beta$-secretase. APP endocytosis can be altered by general modulators of endocytosis, such as dynamin, endophilin and Rab5, or by specifically targeting APP endocytosis, such as by altering the amount and the composition of the APP-FE65-LRP complex. Because $\alpha$-secretase cleavage occurs at or very close to the cell surface, whereas $\beta$-secretase cleavage of wild-type APP mainly occurs in the endosomes, a reduction in APP endocytosis will favor $\alpha$-secretase cleavage, whereas an increase in APP endocytosis will enhance $\beta$-secretase cleavage and $A \beta$ generation. Interestingly, one of the first pathological changes in $\mathrm{AD}$ brain are abnormalities in endosomal morphology [reviewed in 40]. Enlarged endosomal structures are observed long before the onset of the disease and are very similar to the changes seen in cultured cells with experimentally induced increases in endocytosis [21]. 
Together, these findings indicate that alterations in the rate of APP endocytosis may increase $A \beta$ generation not only in cultured cells but also in vivo and may contribute to $\mathrm{AD}$ pathogenesis.

\section{Acknowledgements}

I thank Stephanie Neumann and Susanne Schöbel for critical reading of the manuscript. I am grateful to the Deutsche Forschungsgemeinschaft for financial support through SPP1085/2 (Li862/4-1).

\section{References}

1 Blobel CP: ADAMs: key components in EGFR signalling and development. Nat Rev Mol Cell Biol 2005;6:32-43.

2 Huovila AP, Turner AJ, Pelto-Huikko M, Karkkainen I, Ortiz RM: Shedding light on ADAM metalloproteinases. Trends Biochem Sci 2005;30:413-422.

3 Selkoe DJ, Schenk D: Alzheimer's disease: molecular understanding predicts amyloidbased therapeutics. Annu Rev Pharmacol Toxicol 2003;43:545-584.

4 Citron M: beta-Secretase inhibition for the treatment of Alzheimer's disease - promise and challenge. Trends Pharmacol Sci 2004; 25:92-97.

5 Haass C: Take five-BACE and the gammasecretase quartet conduct Alzheimer's amyloid beta-peptide generation. EMBO J 2004; 23:483-488.

6 Allinson TM, Parkin ET, Turner AJ, Hooper NM: ADAMs family members as amyloid precursor protein alpha-secretases. J Neurosci Res 2003;74:342-352.

7 Skovronsky DM, Moore DB, Milla ME, Doms RW, Lee VM: Protein kinase C-dependent alpha-secretase competes with betasecretase for cleavage of amyloid-beta precursor protein in the trans-golgi network. J Biol Chem 2000;275:2568-2575.

8 Vardy ER, Catto AJ, Hooper NM: Proteolytic mechanisms in amyloid-beta metabolism: therapeutic implications for Alzheimer's disease. Trends Mol Med 2005;11:464-472.

9 Sisodia SS: Beta-amyloid precursor protein cleavage by a membrane-bound protease. Proc Natl Acad Sci USA 1992;89:60756079.

10 Russo C, Venezia V, Repetto E, Nizzari M, Violani E, Carlo P, Schettini G: The amyloid precursor protein and its network of interacting proteins: physiological and pathological implications. Brain Res Brain Res Rev 2005;48:257-264.

11 Neumann S, Schobel S, Jager S, Trautwein A, Haass C, Pietrzik CU, Lichtenthaler SF: Amyloid precursor-like protein 1 influences endocytosis and proteolytic processing of the amyloid precursor protein. J Biol Chem 2006;281:7583-7594

12 Schobel S, Neumann S, Seed B, Lichtenthaler SF: Expression cloning screen for modifiers of amyloid precursor protein shedding. Int J Dev Neurosci 2006;24:141-148.
13 Xu HX, Sweeney D, Greengard P, Gandy S: Metabolism of Alzheimer beta-amyloid precursor protein: Regulation by protein kinase A in intact cells and in a cell-free system. Proc Natl Acad Sci USA 1996;93:40814084.

14 Marambaud P, Chevallier N, Ancolio K, Checler F: Post-transcriptional contribution of a cAMP-dependent pathway to the formation of alpha- and beta/gamma-secretasesderived products of beta APP maturation in human cells expressing wild-type and Swedish mutated beta APP. Mol Med 1998;4:715723.

15 Camden JM, Schrader AM, Camden RE, Gonzalez FA, Erb L, Seye CI, Weisman GA: P2Y2 nucleotide receptors enhance alphasecretase-dependent amyloid precursor protein processing. J Biol Chem 2005;280: 18696-18702.

16 Lee RK, Wurtman RJ, Cox AJ, Nitsch RM: Amyloid precursor protein processing is stimulated by metabotropic glutamate receptors. Proc Natl Acad Sci USA 1995;92: 8083-8087.

17 Reutens AT, Glenn Begley C: Endophilin-1: a multifunctional protein. Int J Biochem Cell Biol 2002;34:1173-1177.

18 Koo EH, Squazzo SL: Evidence that production and release of amyloid beta-protein involves the endocytic pathway. J Biol Chem 1994;269:17386-17389.

19 Chyung JH, Selkoe DJ: Inhibition of receptor mediated endocytosis demonstrates generation of amyloid beta-protein at the cell surface. J Biol Chem 2003;278:51035-51043.

20 Carey RM, Balcz BA, Lopez-Coviella I, Slack BE: Inhibition of dynamin-dependent endocytosis increases shedding of the amyloid precursor protein ectodomain and reduces generation of amyloid beta protein. BMC Cell Biol 2005;6:30.

21 Grbovic OM, Mathews PM, Jiang Y, Schmidt SD, Dinakar R, Summers-Terio NB, Ceresa BP, Nixon RA, Cataldo AM: Rab5-stimulated up-regulation of the endocytic pathway increases intracellular beta-cleaved amyloid precursor protein carboxyl-terminal fragment levels and Abeta production. J Biol Chem 2003;278:31261-31268.

22 King GD, Scott Turner R: Adaptor protein interactions: modulators of amyloid precursor protein metabolism and Alzheimer's disease risk? Exp Neurol 2004;185:208-219.
23 Pietrzik CU, Busse T, Merriam DE, Weggen S, Koo EH: The cytoplasmic domain of the LDL receptor-related protein regulates multiple steps in APP processing. EMBO J 2002; 21:5691-5700.

24 Pietrzik CU, Yoon IS, Jaeger S, Busse T, Weggen $\mathrm{S}$, Koo EH: FE65 constitutes the functional link between the low-density lipoprotein receptor-related protein and the amyloid precursor protein. J Neurosci 2004;24: 4259-4265.

25 Kinoshita A, Whelan CM, Smith CJ, Mikhailenko I, Rebeck GW, Strickland DK, Hyman BT: Demonstration by fluorescence resonance energy transfer of two sites of interaction between the low-density lipoprotein receptor-related protein and the amyloid precursor protein: role of the intracellular adapter protein Fe65. J Neurosci 2001; 21:8354-8361.

26 Trommsdorff M, Borg JP, Margolis B, Herz $\mathrm{J}$ : Interaction of cytosolic adaptor proteins with neuronal apolipoprotein E receptors and the amyloid precursor protein. J Biol Chem 1998;273:33556-33560.

27 Rebeck GW, Moir RD, Mui S, Strickland DK, Tanzi RE, Hyman BT: Association of membrane-bound amyloid precursor protein APP with the apolipoprotein E receptor LRP. Brain Res Mol Brain Res 2001;87:238-245.

28 Herz J, Strickland DK: LRP: a multifunctional scavenger and signaling receptor. J Clin Invest 2001;108:779-784.

29 Ulery PG, Beers J, Mikhailenko I, Tanzi RE, Rebeck GW, Hyman BT, Strickland DK: Modulation of beta-amyloid precursor protein processing by the low density lipoprotein receptor-related protein (LRP). Evidence that LRP contributes to the pathogenesis of Alzheimer's disease. J Biol Chem 2000;275:7410-7415

30 Kummer C, Wehner S, Quast T, Werner S, Herzog V: Expression and potential function of beta-amyloid precursor proteins during cutaneous wound repair. Exp Cell Res 2002; 280:222-232.

31 Beckman M, Iverfeldt K: Increased gene expression of beta-amyloid precursor protein and its homologues APLP1 and APLP2 in human neuroblastoma cells in response to retinoic acid. Neurosci Lett 1997;221:73-76. 
32 Sabo SL, Ikin AF, Buxbaum JD, Greengard P: The amyloid precursor protein and its regulatory protein, FE65, in growth cones and synapses in vitro and in vivo. J Neurosci 2003;23:5407-5415.

33 Sabo SL, Ikin AF, Buxbaum JD, Greengard P: The Alzheimer amyloid precursor protein (APP) and FE65, an APP-binding protein, regulate cell movement. J Cell Biol 2001;153: 1403-1414.

34 Cam JA, Zerbinatti CV, Knisely JM, Hecimovic S, Li Y, Bu G: The low density lipoprotein receptor-related protein $1 \mathrm{~B}$ retains betaamyloid precursor protein at the cell surface and reduces amyloid-beta peptide production. J Biol Chem 2004;279:29639-29646.
35 Andersen OM, Reiche J, Schmidt V, Gotthardt M, Spoelgen R, Behlke J, von Arnim CA, Breiderhoff T, Jansen P, Wu X, Bales KR, Cappai R, Masters CL, Gliemann J, Mufson EJ, Hyman BT, Paul SM, Nykjaer A, Willnow TE: Neuronal sorting protein-related receptor sorLA/LR11 regulates processing of the amyloid precursor protein. Proc Natl Acad Sci USA 2005; 102:13461-13466.

36 Fotinopoulou A, Tsachaki M, Vlavaki M, Poulopoulos A, Rostagno A, Frangione B, Ghiso J, Efthimiopoulos S: BRI2 interacts with amyloid precursor protein (APP) and regulates amyloid beta (Abeta) production. J Biol Chem 2005;280:30768-30772.

37 Matsuda S, Giliberto L, Matsuda Y, Davies P, McGowan E, Pickford F, Ghiso J, Frangione B, D'Adamio L: The familial dementia BRI2 gene binds the Alzheimer gene amyloid-beta precursor protein and inhibits amyloid-beta production. J Biol Chem 2005;280:2891228916.
38 Vidal R, Frangione B, Rostagno A, Mead S, Revesz T, Plant G, Ghiso J: A stop-codon mutation in the BRI gene associated with familial British dementia. Nature 1999;399:776781.

39 Soba P, Eggert S, Wagner K, Zentgraf H, Siehl K, Kreger S, Lower A, Langer A, Merdes G, Paro R, Masters CL, Muller U, Kins S, Beyreuther K: Homo- and heterodimerization of APP family members promotes intercellular adhesion. EMBO J 2005;24:36243634.

40 Nixon RA: Endosome function and dysfunction in Alzheimer's disease and other neurodegenerative diseases. Neurobiol Aging 2005;26:373-382. 